\title{
ESTRATEGIAS BASADAS EN EL ENFOQUE INTERDISCIPLINARIO PARA ABORDAR CONTENIDOS DEL PROGRAMA DE CIENCIAS DE OCTAVO AÑO DE LA EDUCACIÓN GENERAL BÁSICA DE COSTA RICA
}

\author{
STRATEGIES BASED ON THE INTERDISCIPLINARY APPROACH TO TACKLE \\ CONTENT OF THE EIGHTH YEAR SCIENCE PROGRAM OF BASIC GENERAL \\ EDUCATION OF COSTA RICA
}

\section{Diego Leal Aguilar', diegoleal85@yahoo.es Colegio Cooperativo Integral de Coronado, Costa Rica Juan Carlos Solano Mora², solano313@gmail.com Liceo Doctor José María Castro Madriz, Costa Rica Carolina Rojas Oconitrillo ${ }^{3}$, rocaro86@yahoo.es Colegio Los Ángeles P. P. Dominicos, Costa Rica María Isabel Torres Salas ${ }^{4}$, isabeltorresr@yahoo.com Adriana Zúñiga Meléndez ${ }^{5}$, adrianakamu@yahoo.es Universidad Nacional, Costa Rica}

Recibido: 18 de octubre de 2016

Aprobado: 20 de marzo de 2017

\footnotetext{
1 Diego Leal Aguilar, diegoleal85@yahoo.es Profesor de Enseñanza de las Ciencias, Colegio Cooperativo Integral de Coronado. Licenciado en Enseñanza de las Ciencias, Bachiller en Enseñanza de las Ciencias

2 Juan Carlos Solano Mora, solano313@gmail.com Profesor de Enseñanza de las Ciencias, Liceo Doctor José María Castro Madriz. Licenciado en Enseñanza de las Ciencias, Bachiller en Enseñanza de las Ciencias.

${ }^{3}$ Carolina Rojas Oconitrillo, rocaro86@yahoo.es, Profesor de Enseñanza de las Ciencias, Colegio Los Ángeles P. P. Dominicos. Licenciado en Enseñanza de las Ciencias, Bachiller en Enseñanza de las Ciencias.

${ }^{4}$ María Isabel Torres Salas, isabeltorresr@yahoo.com, División de Educología, del Centro de Investigación en Educación, Universidad Nacional, Heredia, Costa Rica

5 Adriana Zúñiga Meléndez, adrianakamu@yahoo.es, Académica de la Escuela de Ciencias Biológicas Universidad Nacional, Heredia, Costa Rica. Dra. en Enseñanza de las Ciencias, Master en Gestión y Licenciada en Enseñanza de las Ciencias.
}

Estrategias basadas en el enfoque interdisciplinario para abordar contenidos del Programa de Ciencias de Octavo Año de la Educación General Básica de Costa Rica.

Diego Leal Aguilar, Juan Carlos Solano Mora, Carolina Rojas Oconitrillo,

María Isabel Torres Salas, Adriana Zúñiga Meléndez

DOI: http://dx.doi.org/10.22458/caes.v8i1.1786

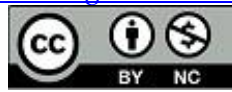




\title{
Resumen
}

El objetivo general de esta investigación fue desarrollar una propuesta metodológica interdisciplinaria para enseñar los contenidos: cambios de la materia, manifestaciones de las reacciones químicas, ley de la conservación de la materia y reacciones endotérmicas y exotérmicas del Programa de Ciencias de octavo año de la Educación General Básica. Para ello se trabajó con una muestra conformada por 9 profesores de ciencias y 60 estudiantes de octavo año, de tres colegios pertenecientes a la Dirección Regional de San José, Costa Rica. De los resultados obtenidos se deriva que, el modelo tradicional es el que más utilizan los docentes, también se evidencia que emplean el enfoque interdisciplinario en sus clases, pero lo hacen de manera esporádica y solo en algunos temas. No obstante, tienen claro que este enfoque les permite a los estudiantes una mayor asimilación de los contenidos y un aprendizaje más contextualizado. Además, se encontró que un alto porcentaje de estudiantes no muestra dificultad en comprender los contenidos de ciencias y que la mayoría de los profesores de la muestra relacionan los contenidos vistos en la clase con la vida diaria, aspecto que probablemente contribuye a que el aprendizaje sea más significativo ya que los estudiantes no encuentren dificultad en los temas desarrollados.

Palabras clave: Estrategias de aprendizaje; interdisciplinariedad; currículo integrado; aprendizaje significativo; modelos de enseñanza.

\begin{abstract}
The overall objective of this research was to develop an interdisciplinary methodological approach to teach content: changes of the material, forms of chemical reactions, law of conservation of matter and endothermic and exothermic reactions of Sciences Program eighth year of basic general education. This work was done with a sample comprised of nine science teachers and sixty students of eighth grade, three schools belonging to the Regional Directorate of San Jose, Costa Rica. From the results it follows that the traditional model is the most used by teachers, also evidence that use an interdisciplinary approach in their classes, But they do so only sporadically and in some subjects. However, are clear that this approach allows students to greater assimilation of the contents and a more contextualized learning. It was also found that a high percentage of students shows no difficulty in understanding the content of science and that most of the teachers in the sample related content viewed in class to everyday life, aspect that probably contributes to make learning more meaningful and could influence students to find no difficulty in developed themes.
\end{abstract}

Keywords: Learning strategies; interdisciplinary; integrated curriculum; meaningful learning; teaching models.

Estrategias basadas en el enfoque interdisciplinario para abordar contenidos del Programa de Ciencias de Octavo Año de la Educación General Básica de Costa Rica.

Diego Leal Aguilar, Juan Carlos Solano Mora, Carolina Rojas Oconitrillo,

María Isabel Torres Salas, Adriana Zúñiga Meléndez.

DOI: http://dx.doi.org/10.22458/caes.v8i1.1786

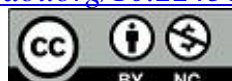

Artículo protegido por licencia Creative Commons 


\section{Referentes conceptuales}

\section{Generalidades sobre Interdisciplinariedad}

En la actualidad, la educación juega un papel fundamental en las diferentes actividades de la sociedad, desde el esclarecimiento de las causas que generan las crisis sociales y la búsqueda de soluciones a las mismas, donde estas logran favorecer al aprendizaje social y la intervención de la ciudadanía en la toma de decisiones a favor del desarrollo social, hasta la aplicación de los conocimientos científicos que propician el desarrollo en los diferentes campos del conocimiento.

Es necesario, por ello que esta educación tenga un abordaje mas integrador que permita establecer una interrelación entre diferentes disciplinas, de forma que se contribuya a desarrollar un proceso de enseñanza y aprendizaje más enriquecedor, con una verdadera cultura general integral; pero sobre todo enfocado a las necesidades que exige la realidad de un contexto globalizado del cual formamos parte. Desde nuestra experiencia, se puede afirmar que son pocos los estudiantes que logran relacionar o integrar los contenidos de las diferentes materias, porque en general, las asignaturas se les han impartido fragmentadas, como si no existiera interrelación entre ellas.

En la actualidad, la mayoría de los sistemas siguen con este gran vacío, que no permite a los estudiantes integrar los saberes aprendidos en el proceso de escolarización,además dejando de lado en su mayoría la realidad del contexto social del cual cada ciudadano es parte. Es decir, hay una desconexión entre lo que se vive y lo que se aprende en las aulas, además se ha caído en la visión fragmentada de los conocimientos; Torres (2000) por su parte dice

[...] que el currículum escolar se ha encontrado desmedidamente desactualizado, alejado de las experiencias vividas por alumnos y alumnas. Muchas de las

Estrategias basadas en el enfoque interdisciplinario para abordar contenidos del Programa de Ciencias de Octavo Año de la Educación General Básica de Costa Rica.

Diego Leal Aguilar, Juan Carlos Solano Mora, Carolina Rojas Oconitrillo,

María Isabel Torres Salas, Adriana Zúñiga Meléndez

DOI: http://dx.doi.org/10.22458/caes.v8i1.1786

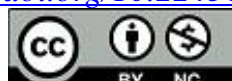


asignaturas están aisladas unas de otras, impidiendo así la construcción y comprensión de nexos que permitan un enlace, entre estas y la realidad (p. 20).

Es por ello, que es evidente la necesidad, que los docentes como actores del proceso educativo tengan una visión de un currículo integrado desde la interdisciplinariedad. Muchas concepciones al respecto establecen que, el enfoque interdisciplinario es una forma integradora de desarrollar el currículo que guiará el proceso de enseñanza y aprendizaje. Por ello Valencia, Méndez, y Jiménez (2008) afirman que la interdisciplinariedad emerge como posibilidad de realizar acciones más integrales y, como una postura crítica frente a las disciplinas que renuncia a una pretensión totalizante. Siguiendo en esta línea, Visser (2002), citado por Grisolía (2008), define interdisciplinariedad como la aplicación de métodos y procedimientos de una disciplina a un problema definido dentro de otra área disciplinaria (p.3).

En síntesis, la interdisciplinariedad permite el intercambio y reforzamiento de varias disciplinas, lo que contribuye a que los estudiantes comprendan de una forma más amplia y a la vez unificada muchos de los conceptos que se van a trabajando en su proceso de enseñanza y aprendizaje.

\section{Interdisciplinariedad en el currículo de las ciencias naturales}

Hoy en día es evidente la importancia que poseen las relaciones interdisciplinarias y la promoción del conocimiento científico, así como la urgente necesidad de implementar estas relaciones dentro del currículo. La enseñanza tradicional que divide, separa, segmenta y atomiza los conocimientos, y pretende que sea el estudiante quien realice integraciones metacognitivas, es cada día más obsoleta, y contribuye menos a preparar al hombre para enfrentar la vida.

Estrategias basadas en el enfoque interdisciplinario para abordar contenidos del Programa de Ciencias de Octavo Año de la Educación General Básica de Costa Rica.

Diego Leal Aguilar, Juan Carlos Solano Mora, Carolina Rojas Oconitrillo,

María Isabel Torres Salas, Adriana Zúñiga Meléndez

DOI: http://dx.doi.org/10.22458/caes.v8i1.1786

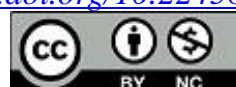


Según Grisolia (2008), uno de los mayores obstáculos que se presentan al pretender un trabajo interdisciplinario lo constituye el aceptar y respetar las diferencias entre las disciplinas involucradas, ya que cada una de las disciplinas posee una especificidad propia, y estas diferencias pueden ser de origen epistemológico, metodológico o semántico. Por otra parte, Lértora (2000) menciona que

[...] en la ejecución de actividades interdisciplinarias se produce la intervención y colaboración entre varias disciplinas, cada una de las cuales tiene unos métodos y un lenguaje propios. Por lo que una dificultad es la confusión que engendra el uso de las mismas palabras con distintas cargas semánticas, determinadas por los diferentes universos discursivos de donde proceden (p.8).

Pero, hay que destacar que, a pesar de la particularidad de cada disciplina, a su vez existen puntos de coincidencia que permiten construir los saberes de manera integrada, dando una visión holística que hace que ese conocimiento cobre sentido. Por eso, resulta de suma importancia que exista disposición para el consenso, y que además se logre establecer un lenguaje en común que facilite la comunicación entre las disciplinas para lograr de manera satisfactoria el trabajo en conjunto, que dé respuesta a una situación en particular.

Para Grisolía (2008), el trabajo interdisciplinario brinda la manera de estudiar los contenidos científicos como aspectos interesantes y de gran valor para la sociedad en la que nos desenvolvemos, y no como un conjunto acumulativo de contenidos aislados, pertenecientes a una única disciplina, que distan de proveer alguna aplicación real y de estar relacionados entre sí.

Resulta innegable que para lograr un adecuado proceso de enseñanza aprendizaje dentro de un modelo interdisciplinario, es vital seguir algunas pautas que permitan el adecuado funcionamiento de esta forma de trabajo, una de estas se basa en el uso de un

Estrategias basadas en el enfoque interdisciplinario para abordar contenidos del Programa de Ciencias de Octavo Año de la Educación General Básica de Costa Rica.

Diego Leal Aguilar, Juan Carlos Solano Mora, Carolina Rojas Oconitrillo,

María Isabel Torres Salas, Adriana Zúñiga Meléndez

DOI: http://dx.doi.org/10.22458/caes.v8i1.1786

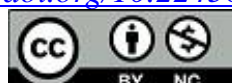


vocabulario, es decir, una semántica uniforme que permita la apropiada comunicación entre las diferentes disciplinas.

Otro punto importante, para la implementación del enfoque interdisciplinario en el aula, es que la formación de los profesionales de la educación incluya preparación en como trabajar con modelos didácticos que estén basados en la interdisciplinariedad; al respecto Grisolia (2008), afirma que no solo es necesaria la preparación de los docentes en las diferentes áreas científicas, sino que es fundamental que los profesores manejen abordajes interdisciplinarios que le permita a los estudiantes una comprensión de las diversas relaciones que existen entre las distintas áreas y, que de esta forma puedan conseguir una visión más integral de los procesos científicos.

\section{Diseño}

En esta investigación, se trabajó con una población de estudiantes de octavo año conformada por tres secciones, pertenecientes a colegios del circuito 01, 05 y 06 de la Regional San José Costa Rica. La muestra se escogió al azar en cada uno de los colegios mencionados, para un total de 60 estudiantes y 9 docentes de ciencias. La investigación se realizó bajo el paradigma naturalista, con un enfoque cualitativo dominante, utilizando el estudio de comunidad. Las categorías de análisis fueron: los modelos de enseñanza utilizados por los docentes y las dificultades que tienen los estudiantes de octavo año en el proceso de aprendizaje de los contenidos: cambios de estado de la materia, manifestaciones de las reacciones químicas, ley de la conservación de la materia, reacciones endotérmicas y exotérmicas, del Programa de Ciencias de Octavo Año. Para la obtención de los datos se trabajó con los siguientes instrumentos: entrevistas de tipo individual en la modalidad semiestructurada, aplicada a los docentes de la muestra, escalas de apreciación para realizar las observaciones a los profesores cuando imparten clases y cuestionarios a los estudiantes.

Estrategias basadas en el enfoque interdisciplinario para abordar contenidos del Programa de Ciencias de Octavo Año de la Educación General Básica de Costa Rica.

Diego Leal Aguilar, Juan Carlos Solano Mora, Carolina Rojas Oconitrillo,

María Isabel Torres Salas, Adriana Zúñiga Meléndez

DOI: http://dx.doi.org/10.22458/caes.v8i1.1786

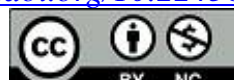




\section{Resultados y discusión}

Los resultados encontrados en la investigación, con respecto a los principales modelos educativos utilizados por los docentes durante el desarrollo de las lecciones de ciencias, indican que éstos aplican distintos modelos en su proceso de enseñanza, pero que el tradicional es el que más emplean como se muestra en la tabla 1, donde se puede ver que hay un predominio por el uso de este modelo.

Tabla 1: Opinión de los docentes de los colegios en estudio sobre la implementación de los modelos educativos en el desarrollo de las lecciones de ciencias.

\begin{tabular}{cc}
\hline Pregunta 1. & $\begin{array}{l}\text { Modelos educativos que se utilizan comúnmente en el } \\
\text { desarrollo de las lecciones de ciencias }\end{array}$ \\
\hline $\begin{array}{c}\text { Docentes } \\
\text { Circuito 01 }\end{array}$ & $\begin{array}{c}\text { Resumen de las ideas principales } \\
\text { mayor proporción y el abordaje interdisciplinario. }\end{array}$ \\
Circuito 05 & - $\quad \begin{array}{l}\text { Enseñanza tradicional en primer lugar y enseñanza por } \\
\text { indagación, en conjunto con un abordaje interdisciplinario. } \\
\text { Circuito 06 }\end{array}$ \\
\end{tabular}

Nota: Entrevista a los docentes de los colegios pertenecientes al circuito 01, 05, 06

Es importante destacar que el modelo conductista -que en nuestros medios se le denomina como tradicional- sigue estando presente de forma predominante, a pesar del auge de nuevos modelos y paradigmas educativos en la sociedad actual, y pese al debate existente donde se discute que los cambios acelerados, la globalización y la influencia de las tecnologías de la información obligan a un cambio urgente.

Estrategias basadas en el enfoque interdisciplinario para abordar contenidos del Programa de Ciencias de Octavo Año de la Educación General Básica de Costa Rica.

Diego Leal Aguilar, Juan Carlos Solano Mora, Carolina Rojas Oconitrillo, María Isabel Torres Salas, Adriana Zúñiga Meléndez DOI: http://dx.doi.org/10.22458/caes.v8i1.1786

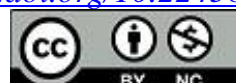


El modelo tradicional está relacionado con los procesos de aprendizaje que tienen un enfoque conductista, y las estrategias de enseñanza apuntan principalmente a la transmisión de conocimiento. En este modelo el profesor dirige el proceso, él es quién organiza el conocimiento y dice a sus alumnos lo que deben aprender y éstos actúan como receptores. Según Godemann (2007), bajo este modelo el estudiante es despojado de su responsabilidad, de su libertad y de su dignidad, para ser "moldeado" por aquellos que utilicen efectivamente las herramientas del conductismo.

Sin embargo, a pesar de utilizar mayoritariamente el modelo tradicional, también se evidencia en las opiniones de los docentes (tabla1), que en sus clases emplean el enfoque interdisciplinario en el abordaje de algunas temáticas, pero que lo hacen ocasionalmente $y$, justifican que depende del tema en estudio, ya que muchos contenidos son muy factuales y abstractos, lo que impide ese tipo de desarrollo, aspecto que más bien debería motivarlos a utilizar este enfoque para dar una visión más integral de los contenidos enseñados.

Por otra parte, ellos expresan en la entrevista que es importante llevar a los estudiantes a que contextualicen sus conocimientos y aprendan de manera holística, que es quizás uno de los fundamentos por los que se rige la interdisciplinariedad, el de unificar los diferentes saberes en pro de un proceso de aprendizaje no segmentado. En este sentido, Grisolia (2008) expresa que el enfoque interdisciplinario permite obtener una visión más amplia, completa y unificada de un problema, así como conllevar a la obtención de una solución más integral y adecuada al sistema en estudio.

De acuerdo con lo anterior, se puede afirmar que a pesar de que los docentes utilizan en el desarrollo de sus clases de forma predominante el modelo tradicional, también utilizan

Estrategias basadas en el enfoque interdisciplinario para abordar contenidos del Programa de Ciencias de Octavo Año de la Educación General Básica de Costa Rica.

Diego Leal Aguilar, Juan Carlos Solano Mora, Carolina Rojas Oconitrillo, María Isabel Torres Salas, Adriana Zúñiga Meléndez DOI: http://dx.doi.org/10.22458/caes.v8i1.1786 
otros enfoques y tienen conciencia de la importancia de éstos en la enseñanza de las Ciencias Naturales.

En este mismo sentido, se le consultó a los estudiantes si los contenidos de química desarrollados en clase, son relacionados por el profesor con otras ciencias como la biología y la física, al respecto en el gráfico 1 se puede ver que las opiniones están muy divididas, siendo los estudiantes del circuito 06 los que, en un porcentaje mayor opinaron que "siempre" lo utiliza. Sin embargo, se puede decir que "pocas veces" ocupa porcentajes considerables, quedando al descubierto que el abordaje interdisciplinario se hace de forma esporádica. Esta percepción por parte de los estudiantes es congruente con lo expresado por los docentes, quienes afirman utilizar este enfoque en sus lecciones cada vez que el tema lo permita, es decir de manera ocasional.

Estrategias basadas en el enfoque interdisciplinario para abordar contenidos del Programa de Ciencias de Octavo Año de la Educación General Básica de Costa Rica.

Diego Leal Aguilar, Juan Carlos Solano Mora, Carolina Rojas Oconitrillo,

María Isabel Torres Salas, Adriana Zúñiga Meléndez.

DOI: http://dx.doi.org/10.22458/caes.v8i1.1786 
Gráfico 1. Apreciación de los estudiantes, en cuanto a si los contenidos de química desarrollados en clase, son relacionados por el profesor con otras ciencias como la biología y la física.

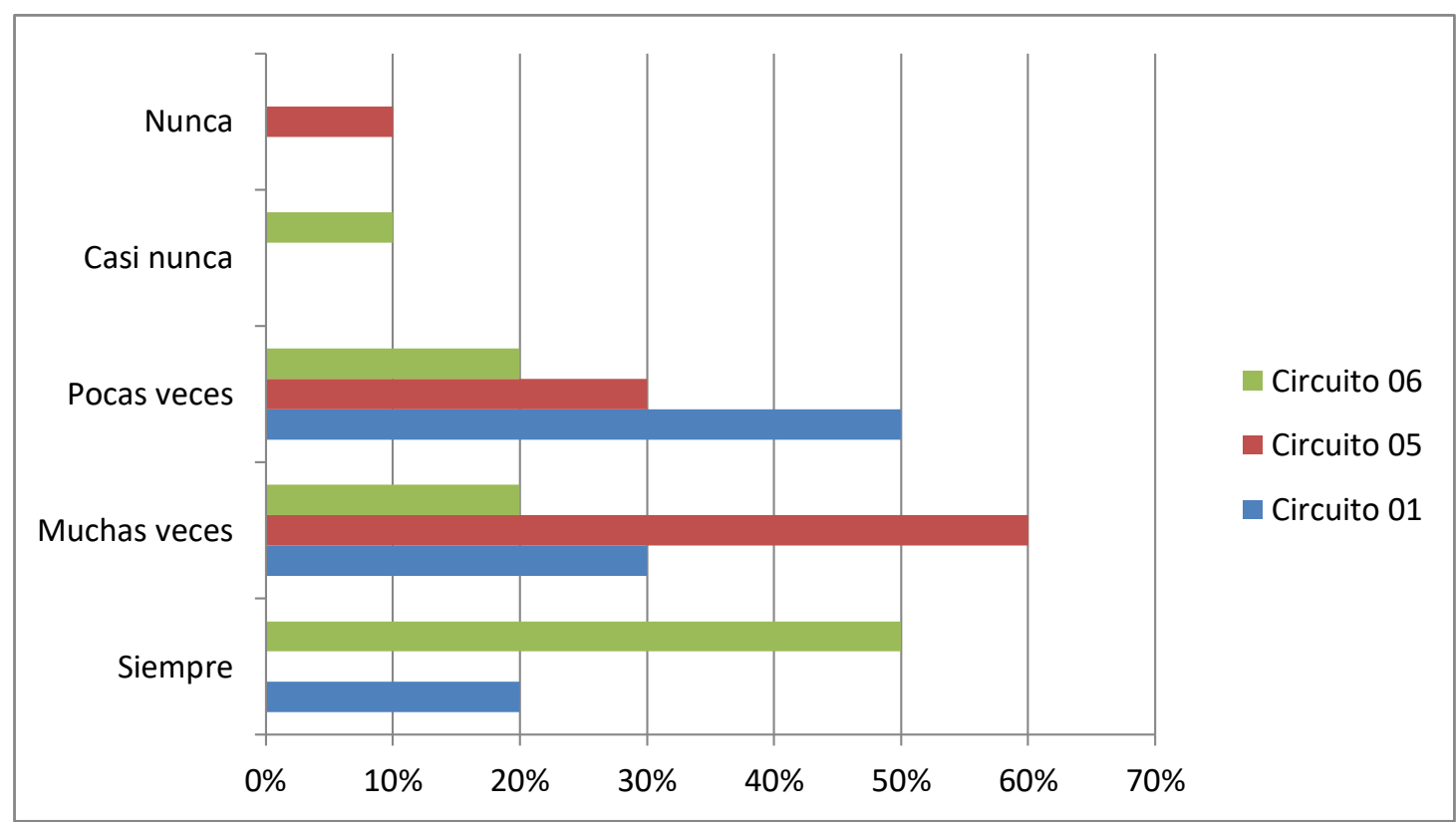

Nota: Entrevista a los docentes de los colegios pertenecientes al circuito 01, 05, 06.

Además, es importante recalcar, que al observar las clases también se pudo corroborar que el abordaje interdisciplinario se realiza a veces, además pareciera que es utilizado de forma empírica, ya que no se desarrollan estrategias precisas de este tipo de enfoque en el trabajo de aula, aunque si se rescata la correlación que se hace con las otras ciencias en el desarrollo de un tema determinado.

El abordaje de los contenidos desde varias áreas del conocimiento es fundamental para que el alumno comprenda los conceptos de una forma integrada y no de manera atomizada, lo que impide en la mayoría de los casos relacionar lo aprendido con el contexto en el que está inmerso. De ahí que, la enseñanza de las Ciencias Naturales

Estrategias basadas en el enfoque interdisciplinario para abordar contenidos del Programa de Ciencias de Octavo Año de la Educación General Básica de Costa Rica.

Diego Leal Aguilar, Juan Carlos Solano Mora, Carolina Rojas Oconitrillo,

María Isabel Torres Salas, Adriana Zúñiga Meléndez

DOI: http://dx.doi.org/10.22458/caes.v8i1.1786

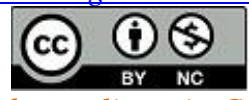


planteada desde el enfoque interdisciplinario puede ofrecer nuevas y diversas oportunidades para el logro de mejores aprendizajes.

También se consideró la percepción de los docentes sobre si ellos relacionan los temas de ciencias con hechos de la vida cotidiana; al respecto evidencian que la mayoría del tiempo relacionan lo visto en clase con los hechos que ocurren en la cotidianeidad, con el fin de contextualizar los temas desarrollados en el aula, así como despertar el interés del alumnado por los mismos (tabla 2).

En esta misma línea el $100 \%$ de los estudiantes afirman que los contenidos de ciencias desarrollados en clase son útiles en el hogar, la comunidad y en la vida cotidiana, es decir, ellos consideran que lo aprendido en clases es útil para la vida. Lo cual denota que el proceso mediado por sus docentes está orientado hacia un aprendizaje aplicado a la vida, a pesar del uso del modelo tradicional en forma predominante.

Es importante destacar que es necesaria la existencia de una relación entre lo que enseñamos y el contexto del cual es parte el estudiante, para que el aprendizaje tenga el impacto deseado; ya que la relación que podamos establecer entre lo aprendido a priori y lo que se vivencia, es un factor que contribuye a que el estudiante encuentre sentido a lo que aprende. En esta misma línea, Sánchez (2003) afirma que "[...] el aprendizaje significativo es el resultado de la interacción de los conocimientos previos y los conocimientos nuevos y de su adaptación al contexto, y que además va a ser funcional en determinado momento de la vida del individuo" (El aprendizaje Significativo, párr. 4)

Estrategias basadas en el enfoque interdisciplinario para abordar contenidos del Programa de Ciencias de Octavo Año de la Educación General Básica de Costa Rica.

Diego Leal Aguilar, Juan Carlos Solano Mora, Carolina Rojas Oconitrillo, María Isabel Torres Salas, Adriana Zúñiga Meléndez. DOI: http://dx.doi.org/10.22458/caes.v8i1.1786 
Tabla 2. Opinión de los docentes sobre si relacionan los temas de ciencias con hechos de la vida cotidiana de los estudiantes, durante el desarrollo de sus lecciones.

Pregunta 9 ¿Al desarrollar los temas de ciencias usted los relaciona con hechos de la vida cotidiana de los estudiantes? ¿Con qué frecuencia?

\section{Docentes}

Circuito 01

Circuito 05

Circuito 06

\section{Resumen de las ideas principales}

- Cada vez que el contenido lo permita, la mayoría de las veces

- Si, con aquellos temas fáciles de contextualizar y relacionar.

- En la mayoría de los casos, intenta hablarles de noticias sobre avances científicos y su aplicación en la vida y esto despierta el interés en los alumnos.

Nota: Entrevista a los docentes de los colegios pertenecientes al circuito 01, 05, 06

Otro factor importante es que sí el estudiante relaciona los conceptos vistos en clase con la cotidianidad, lo que aprende va tener significado y esto puede despertar el interés por la materia; entonces la consigna parece estar en crear vínculos entre lo que el alumno sabe, ya previamente, y lo que está por aprender de manera inmediata, con el fin de que alcance un aprendizaje significativo, como lo menciona Sánchez (2003), “[...] el aprendizaje significativo es el resultado de la interacción de los conocimientos previos y los conocimientos nuevos y de su adaptación al contexto, y que además va a ser funcional en determinado momento de la vida del individuo (El aprendizaje significativo, párr. 5).

En todo proceso de aprendizaje es esencial que los individuos conozcan y descubran su propia realidad, que indaguen el porqué, el cómo y el para qué aprenden, es decir deben

Estrategias basadas en el enfoque interdisciplinario para abordar contenidos del Programa de Ciencias de Octavo Año de la Educación General Básica de Costa Rica.

Diego Leal Aguilar, Juan Carlos Solano Mora, Carolina Rojas Oconitrillo,

María Isabel Torres Salas, Adriana Zúñiga Meléndez

DOI: http://dx.doi.org/10.22458/caes.v8i1.1786

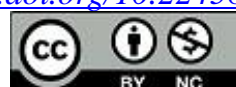


ir más allá de lo que dicen los libros de texto y de lo que "ya saben". Pero para ello la mediación pedagógica que realiza el docente debe estar dirigida no solo al aprendizaje de contenidos, sino también a descubrir la información necesaria que conlleve a aprendizajes significativos, para desarrollar un proceso que les permita relacionar lo que han aprendido, con las vivencias y medios que los rodea.

Por lo tanto, el educador tiene el deber de favorecer procesos de aprendizaje enriquecedores, facilitando las herramientas necesarias para que el alumno por sí mismo pueda descubrir y aprender de manera significativa. Según Bruner, citado por Zarza (2009), “[...] la condición indispensable para aprender una información de manera significativa, es tener la experiencia personal de descubrirla, es decir, el descubrimiento fomenta el aprendizaje significativo" (p.8).

En síntesis, en lo que respecta a los modelos de enseñanza utilizados por los docentes se podría afirmar que prevalece mayoritariamente el modelo tradicional, lo que evidencia que el conductismo sigue dejando huella en cada uno de los individuos que han pasado por su sistema, no permitiendo en parte, ir más allá de la mera transmisión de contenidos. Sin embargo, los docentes también utilizan el enfoque interdisciplinario, aunque en menor escala y opinan de manera unánime que la implementación de estrategias desde este enfoque es muy importante en el desarrollo sus lecciones, pues consideran que permite al estudiante una mejor compresión de los temas, al mostrarlo desde una visión holística y relacionado con su entorno.

Por otra parte, al indagar sobre la categoría de análisis (y las) dificultades que presentan los estudiantes en el aprendizaje de los temas del Programa de Ciencias de Octavo Año, los docentes consideran que los contenidos en los que presentan mayor dificultad los estudiantes son: la tabla periódica y sus características, nomenclatura y reacciones

Estrategias basadas en el enfoque interdisciplinario para abordar contenidos del Programa de Ciencias de Octavo Año de la Educación General Básica de Costa Rica.

Diego Leal Aguilar, Juan Carlos Solano Mora, Carolina Rojas Oconitrillo,

María Isabel Torres Salas, Adriana Zúñiga Meléndez

DOI: http://dx.doi.org/10.22458/caes.v8i1.1786

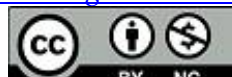


químicas. Esto, según su percepción, es debido a que estos contenidos son abstractos, lo que lleva a que muchos estudiantes no los comprendan y opten por una actitud de desinterés. No obstante, al consultarle a los estudiantes cuáles temas se les dificultan, la mayoría expresa que no tiene problemas para entender y aprender la materia, ya que, los docentes explican de manera que facilita su proceso de aprendizaje.

Se puede afirmar que, un alto porcentaje de estudiantes no muestra dificultad en comprender los contenidos dados por el docente en el aula; a pesar de que se evidenció que el modelo educativo más utilizado por los docentes es el tradicional, donde ellos casi no tienen participación en la construcción de su conocimiento. Lo anterior quizás se deba, entre otros aspectos, a que el estudiantado ha llegado a un nivel de adaptación de este modelo como afirma Ruiz, Castaño y Baronat (1999) "[...] en la enseñanza de las ciencias ha dominado un planteamiento basado en la transmisión de conocimientos: el profesor elabora contenidos que el alumno recibe pasivamente, muchas veces con indiferencia" (p.84).

Es importante destacar que, aunque la mayoría de estudiantes dice no tener dificultades de aprendizaje, hay algunos que opinan que tienen dificultad, porque su forma de aprender no es la misma que utiliza el docente para explicar, factor que es necesario tomar en consideración, para escoger las estrategias y metodologías al planear las clases, ya que no deja de ser una variante que influye directamente en el proceso de enseñanza y aprendizaje. Donde lo planeado no coincide muchas veces con lo llevado a la práctica. En relación con lo anterior, Torres (2000) afirma "[...] que la coherencia con que se dice que se planifican los contenidos de los sistemas educativos, es difícilmente visible por el alumnado e incluso en ocasiones, por el propio cuerpo docente" (p. 29).

Estrategias basadas en el enfoque interdisciplinario para abordar contenidos del Programa de Ciencias de Octavo Año de la Educación General Básica de Costa Rica.

Diego Leal Aguilar, Juan Carlos Solano Mora, Carolina Rojas Oconitrillo, 
En síntesis, se puede afirmar que existen diferentes factores que llevan a un educando a tener dificultades en su proceso de aprendizaje, dentro de los que se encuentran la'

desmotivación, problemas de actitud frente a la materia, indisciplina temas desvinculados de la realidad, entre otros, pero en términos generales a los estudiantes de la muestra analizada muy pocas veces se les dificultó comprender los contenidos desarrollados en clase, tal como lo indican ellos y los docentes.

\section{Conclusiones}

Abordar la interdisciplinariedad en los procesos educativos supone entre otras cosas que seamos capases de dimensionar los aportes de este enfoque educativo sobre el aprendizaje.Este enfoque que posibilita la formación integral e integradora de aprendizajes que lo transforman en una muy buena herramienta para la formación de individuos competentes.

Por esto conocer si este enfoque es utilizado por los docentes, así como la concepción que tienen del mismo, resulta fundamental si la preocupación es mejorar la calidad de la educación que se ofrece. A la luz de estos planteamientos es que el presente estudio tuvo como eje central estudiar las prácticas pedagógicas y a su vez diseñar estrategias que favorecieran la interdisciplinariedad. En este sentido al analizar los resultados obtenidos respecto a la práctica de los docentes se puede constatar que los mismos, solo en algunas ocasiones, utilizan un enfoque interdisciplinario, que normalmente se encuentra asociado al contenido que está desarrollando, más que a una verdadera visión de la planificación interdisciplinar.

De igual forma a pesar de que consideran que la interdisciplinariedad resulta una buena herramienta para lograr que los estudiantes asimilen de mejor forma los aprendizajes de

Estrategias basadas en el enfoque interdisciplinario para abordar contenidos del Programa de Ciencias de Octavo Año de la Educación General Básica de Costa Rica.

Diego Leal Aguilar, Juan Carlos Solano Mora, Carolina Rojas Oconitrillo,

María Isabel Torres Salas, Adriana Zúñiga Meléndez

DOI: http://dx.doi.org/10.22458/caes.v8i1.1786

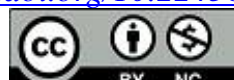


las ciencias, las planificaciones y desarrollo de sus lecciones sigue estando muy sesgada hacia el modelo tradicional de enseñanza.

Por otra parte, aun cuando los estudiantes manifestaron que los profesores relacionan los contenidos vistos en la clase con la vida diaria, aspecto que según ellos hace que el aprendizaje sea más significativo y fácil, sigue quedando vago el tratamiento de los contenidos desde el enfoque interdisciplinar.

Además, se logró determinar, que el abordaje de la mayoría de los temas del programa de estudio de octavo se hace con un enfoque disciplinario, por lo que se ve la necesidad de hacer una propuesta didáctica interdisciplinaria que le permita al estudiante tener un conocimiento holístico acerca de diferentes temas de las ciencias. Por ello, se construyó una propuesta donde los contenidos: estados de la materia, reacciones químicas, ley de conservación de la materia y reacciones endotérmicas y exotérmicas se desarrollan con un abordaje interdisciplinar, estableciendo relaciones desde la química, la física y la biología de una forma holística y no de forma fragmentada como generalmente se enseñan.

Finalmente, si se considera que la interdisciplinariedad busca unificar los diferentes saberes en pro de un proceso de aprendizaje no segmentado, que lleve a los estudiantes a contextualizar sus conocimientos y aprender significativamente; diseñar estrategias que faciliten su implementación en el aula debería de transformarse en una tarea constante de la mediación pedagógica.

\section{Recomendaciones}

Se recomienda a los docentes: incluir en sus planeamientos y prácticas pedagógicas, estrategias de corte interdisciplinar para que los estudiantes perciban los fenómenos de la

Estrategias basadas en el enfoque interdisciplinario para abordar contenidos del Programa de Ciencias de Octavo Año de la Educación General Básica de Costa Rica.

Diego Leal Aguilar, Juan Carlos Solano Mora, Carolina Rojas Oconitrillo,

María Isabel Torres Salas, Adriana Zúñiga Meléndez

DOI: http://dx.doi.org/10.22458/caes.v8i1.1786

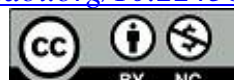


naturaleza y en general los hechos de la vida diaria como un todo y no como conocimientos fragmentados, es decir, de forma holística y cercana a su realidad tanto natural como social, para que logren contextualizar los diversos temas y se promueva un aprendizaje para la vida.

Por otra parte, al Ministerio de Educación Pública se le recomienda promover el desarrollo de estrategias didácticas desde un enfoque interdisciplinario, desarrollando políticas educativas que permitan la capacitación permanente de los docentes y utilizar la propuesta generada como resultado de esta investigación.

\section{Referencias}

Godemann, J. (2007). Métodos de enseñanza y aprendizaje interdisciplinario. Polis : revista académica de la Universidad Bolivariana, ISSN 0717-6554,(16). pp.1-27. Recuperado dehttp://dialnet.unirioja.es/servlet/revista [ 28 de marzo 2014]

Grisolía, M. (2008). La interdisciplinariedad en la enseñanza de las ciencias (interdisciplinarity in science teaching. Ciencia \& Educacao, 11, 1-11. Recuperado de http://www2.fc.unesp.br/cienciaeeducacao/ [Consulta 10 marzo, 2015]

Lértora, C. (2000). Enfoque epistemológico de los problemas de interdisciplinariedad. Polylog, Foro para filosofía intercultural, p. 1. Recuperado de http://them.polylog.org/1/alc-es.htmç/ [Consulta 25 abril, 2014]

Ruiz, E., Castaño, N. y Baronat, N. (1999). Reflexiones sobre el enfoque interdisciplinar y su proyección práctica en la formación del profesorado. Revista electrónica interuniversitaria de formación del profesorado, 2(1), 270-276 Recuperado de http://aufop.com/aufop/uploaded_files/articulos/1224327955.pdf/ [Consulta mayo, 2014]

Sánchez, M. (2003). El aprendizaje Significativo. Psicopedagogía. Recuperado de http://www.psicopedagogia.com/definicion/aprendizaje\%20significativo/ [Consulta 20 enero, 2012]

Estrategias basadas en el enfoque interdisciplinario para abordar contenidos del Programa de Ciencias de Octavo Año de la Educación General Básica de Costa Rica.

Diego Leal Aguilar, Juan Carlos Solano Mora, Carolina Rojas Oconitrillo,

María Isabel Torres Salas, Adriana Zúñiga Meléndez.

DOI: http://dx.doi.org/10.22458/caes.v8i1.1786

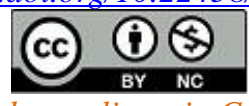


Torres, J. (2000). Globalización e interdisciplinariedas: el curriculum integrado. España: Ediciones Morata.

Valencia, S., Méndez, O. y Jiménez, G. (2008). ¿Enseñanza de las ciencias por disciplinas o interdisciplinariedad en la escuela?, 23, 78-88. Profesores Universidad Pedagógica Nacional, Bogotá, Colombia.

Visser, J. (2002). Innovación: necesidad científica y elección artística. Ponencia presentada en el marco de la inauguración de las "Cátedras de Innovación Educativa" de la Coordinación General del Sistema para la Innovación del Aprendizaje, Universidad de Guadalajara, Guadalajara, México, 2002. Recuperado de

http://www.learndev.org/dl/Innovacion-UdG-2002.pdf/ [Consulta 10 marzo, 2008]

Zarza, O. (mayo, 2009). Aprendizaje por Descubrimiento. Revista digital: Innovación y experiencias educativas. DEP. LEGAL: GR2922/2007, 18, 1-11. Recuperado de http://www.csicsif.es/andalucia/modules/mod ense/revista/pdf/Numero 18/OLGA ZARZA CORTES01.pdf/ [Consulta 15 jun. 2012]

Estrategias basadas en el enfoque interdisciplinario para abordar contenidos del Programa de Ciencias de Octavo Año de la Educación General Básica de Costa Rica.

Diego Leal Aguilar, Juan Carlos Solano Mora, Carolina Rojas Oconitrillo,

María Isabel Torres Salas, Adriana Zúñiga Meléndez.

DOI: http://dx.doi.org/10.22458/caes.v8i1.1786 